Review

\title{
The effect of combined oral contraceptives and hormone therapy during assisted reproductive technologies on the function of the hemostatic system in non-pregnant and pregnant women
}

\author{
Evgeniya V. Kirakosyan, Elena A. Sosnova
}

I.M. Sechenov First Moscow State Medical University (Sechenov University), Moscow, Russia

Received 4 February 2020, Revised 15 July 2020, Accepted 25 October 2020

(C) 2020, Kirakosyan E.V., Sosnova E.A.

(C) 2020, Russian Open Medical Journal

\begin{abstract}
A review of world literature was conducted in the databases Scopus, Web of Science, MedLine, Cochrane CENTRAL, Cochrane Database of Systematic Reviews (CDSR), Database of Abstracts of Reviews of Effectiveness (DARE), EMBASE, Global Health, CyberLeninka, $\mathrm{RSCl}$ on the problem of the effect of combined oral contraceptives (COCs) and hormone therapy during assisted reproductive technologies on the function of the hemostatic system in non-pregnant and pregnant women. The scientific concept of this work is different in that we have summarized and analyzed information on the effect of sex hormones entering the woman's body from the outside on individual links and the hemostatic system as a whole, determined the relationship between drug administration and thrombotic complications, optimized the sequence of diagnosis of conditions predisposing to thrombosis. The scientific novelty of this work is that we have compared the effects of COCs and hormonal changes during pregnancy on the hemostatic system and found that changes in the hemostatic system in women taking COCs are similar to those that occur during pregnancy.
\end{abstract}

Keywords: COC; hemostasis; thrombosis; pregnancy; IVF

Cite as Kirakosyan EV, Sosnova EA. The effect of combined oral contraceptives and hormone therapy during assisted reproductive technologies on the function of the hemostatic system in non-pregnant and pregnant women. Russian Open Medical Journal 2021; 10 : e0210.

Correspondence to Evgeniya Kirakosyan. Address: I.M. Sechenov First Moscow State Medical University (Sechenov University), st. Trubetskaya, 8, p. 2, Moscow, 119991, Russia. Phone: +79165747963. E-mail: evgeniya.kirakosyan@mail.ru.

\section{Physiological effects of estrogen and progesterone on the} hemostatic system

From the moment the first combined oral contraceptives (COCs) appeared to date, thrombotic complications have been on the first place in the list of side effects of this group of drugs. Most often, deep vein thrombosis of the lower extremities (65\%), iliofemoral thrombosis (35\%), pulmonary embolism (9\%) occur [1]. All these years, studies have been conducted to determine the effect of COCs on the hemostatic system and their role in the pathogenesis of iatrogenic complications associated with impaired blood coagulation.

The scientific novelty of this work is that we have compared the effects of COCs and hormonal changes during pregnancy on the hemostatic system and found that changes in the hemostatic system in women taking COCs are similar to those that occur during pregnancy. There are currently no modern review publications precisely affecting this significantly important topic.

The scientific concept of this work is different in that we have summarized and analyzed information on the effect of sex hormones entering the woman's body from the outside on individual links and the hemostatic system as a whole, determined the relationship between drug administration and thrombotic complications, optimized the sequence of diagnosis of conditions predisposing to thrombosis.
It has been established that estrogens, depending on the dose, increase the concentration and activity of I, II, VII, X and XII coagulation factors, lower the level of antithrombin III (AT III) - a natural anticoagulant [1]. Also, different drugs of the progesterone group have an effect on hemostasis [2].

A large study in 2009 showed a five-fold increase in the risk of venous thrombosis in women taking COCs compared with women not taking COCs (control group). The risk varied depending on the type of progestogen: the use of COCs containing levonorgestrel (LNG) resulted in a four-fold increase in the risk of venous thrombosis compared to the control group; for COCs containing gestoden, the risk was 5.6 times higher, desogestrel -7.3 times, cyproterone acetate -6.8 times, drospirenone (DRSP) -6.3 times compared with the control group. The risk of venous thrombosis was also associated with a dose of estrogen $[3,4]$.

The historical nationwide register-based cohort study in the United States for 10 years assessed the risk of venous thromboembolism (VTE) when using COCs depending on the type of progestogen and estrogen dose in 4246 women. The incidence of VTE in the control group was 3.7 per 10,000 women per year. The risk of VTE in women taking COCs with LNG $+30 \mu \mathrm{g}$ ethinylestradiol (EE) was 2.19, with LNG + 30-40 $\mu \mathrm{g} \mathrm{EE} \mathrm{-} \mathrm{2.28.} \mathrm{The}$ risk of VTE when taking $\mathrm{COC}$ with $30 \mu \mathrm{gEE}+$ desogestrel was 4.21, with $30 \mu \mathrm{g} \mathrm{EE}+$ gestoden - 4.23, with $30 \mu \mathrm{g} \mathrm{EE} \mathrm{+} \mathrm{DRSP} \mathrm{-} \mathrm{4.47.}$ Corresponding estimates of COCs with $20 \mu \mathrm{g} \mathrm{EE}+$ the same 
progestogens were $3.26,3.50$ and 4.84 . Only drugs containing pure progestogens did not increase the risk of VTE, regardless of whether they were taken as tablets with a low dose of norethisterone, or tablets with desogestrel, or in the form of hormonal releasing systems $[5,6]$.

In a German case-control study, the risks of VTE were evaluated using a new generation of COCs with DRSP and dienogest (DNG). Among 60,000 European women taking COCs with DRSP, there was no evidence of an increased risk of VTE compared with women taking LNG [7]. In another large cohort study involving 67,000 women, no evidence of an increased risk of VTE was found among women taking COCs with DRSP, compared with women taking COCs with other progestogens, including LNG. Next, 680 cases of VTE and a comparison with 2720 women from the control group were analyzed. The study confirmed an increased risk of thromboembolic complications in women taking COCs [8]. Comparison of DNG / EE and DRSP / EE with other lowdose COCs, including LNG / EE, showed no increased risk of VTE for drugs containing DNG and DRSP.

Hormonal contraceptives have gone a long evolutionary path, as a result of which the dose of estrogen has decreased and the quality of gestagens in the composition of COCs has improved. Thus, the number of cases of thrombotic complications while taking COCs has significantly decreased, but still remains the number 1 problem in this field of medicine.

\section{Changes in the hemostatic system while taking COC}

It has now been established that the most important cause of thrombotic complications is the initial, sometimes hidden, disorders of the hemostatic system, predisposing to increased blood coagulation and thrombosis. COCs, even with a low content of estrogen component, can be dangerous in conditions of genetic or acquired thrombophilia, circulation of antiphospholipid antibodies, hyperhomocysteinemia. Unfortunately, so far, these important risk factors are not always taken into account when prescribing this method of contraception.

It is known that women with various forms of thrombophilia are more likely to experience various complications of pregnancy (anembryonia, early and late miscarriages, premature detachment of a normally located placenta, preeclampsia, VTE). Considering the unidirectional effect of COCs and genetic thrombophilia on the hemostatic system, it is COCs in many situations that are a trigger factor in the development of thrombotic complications in case of already existing imbalances in the pro- and anticoagulant mechanisms.

That is why one of the main stages of the examination of patients is: conducting special studies in the presence of thrombosis in the history of taking $\mathrm{COC}$, as well as possible changes in thrombotic hemostasis in the absence of clinical manifestations, determining the relationship between COC taking and the state of the hemostatic system and predicting possible pregnancy complications.

Recent data on hereditary and acquired forms of thrombophilia have made it possible to rethink the causes and pathogenetic mechanisms of thrombotic complications, including during the use of COCs. Thrombophilia - hereditary or acquired defects in the hemostatic system, predisposing to increased blood clotting and thrombosis. Mutations and polymorphisms of genes differ in prevalence in the population, mechanisms and degree of influence on the blood coagulation system [9].
The most common in the population are polymorphisms of the plasminogen activator inhibitor type 1 (PAl-1) - 26\% in the homozygous and $50 \%$ in the heterozygous version, factor I fibrinogen (7\%) and factor XIII [10,11]. Further prevalence is followed by folate cycle gene mutations (MTHFR C677T, MTAC, MTTR, MTR) and platelet unit of the hemostatic system [12]. The mutation of factor $\mathrm{V}$ Leiden is relatively rare, but extremely important: it increases the risk of thrombosis in heterozygous carriers by 3-8 times, in homozygous carriers, by $50-80$ times in comparison with the general population [13]. When taking COCs, the risk of VTE increases by 35-40 in heterozygotes and by 80-100 times in homozygotes compared to healthy women who do not use COCs [14]. It should be remembered about the oftenencountered combined forms of thrombophilia: a combination of acquired (antiphospholipid syndrome) and genetic / multigenic forms [15].

Thus, most mutations and polymorphisms of hemostatic system genes may not occur spontaneously throughout life. Often, a combination of several mutations or additional risk factors for thrombotic complications (taking COCs, pregnancy, surgical interventions, trauma, prolonged immobilization, smoking, infectious and oncological diseases, etc.) can provoke a manifestation of the disease. It is noteworthy that in women with thrombotic complications during the first months of taking COCs, hereditary hemostatic disorders are much more often detected than in women with thrombotic complications with a longer intake.

It is necessary to consider the effect of COCs on individual parts of the hemostatic system.

COCs affect platelet function, leading to their hyperactivity, the degree of which depends on the duration of the drug. Markers of platelet reactions increase in blood plasma: P-thromboglobulin (P-TG), 4-antiheparin platelet factor (PF4), platelet factor 3 (PF3) and thromboxane $\mathrm{A} 2$ ( $\mathrm{T} \times \mathrm{A} 2$ ). Many authors believe that in the process of taking COCs, moderate platelet activation is observed that does not require additional therapy, but taking these drugs for more than 9 months leads to progression of platelet aggregation due to the initiation of blood coagulation through the platelet link [16]. Platelet reactions are the first sign of activation of the hemostatic system, and in the presence of a genetic or acquired form of thrombophilia, the patient may initially have platelet hyperaggregation before taking COCs.

When prescribing COCs, the aggregation and functional activity of platelets should be checked initially and in dynamics at intervals of 2-3 months. If initially the aggregation parameters are normal, then there are no contraindications for the appointment of COCs on this side. If platelet hyperaggregation develops while taking COCs, it is necessary to prescribe antiplatelet agents for the entire period of contraception with mandatory dynamic control.

A persistent increase in the level of PAI- 1 and, consequently, a decrease in the concentration of tissue plasminogen activator (tPA) and plasminogen indicate the effect of COCs on the process of fibrinolysis. At the initial normal concentration of PAI-1 in the blood, by the third month of taking COCs, an increase in its level by 2-3 times is observed, and by the 9th month the maximum values of PAl-1 are recorded [10]. Changes in the fibrinolysis system are also indicated by an increase in the concentration of fibrin degradation products (FDP) - D-dimer and plasmin-antiplasmin (PAP) complexes [17]. Thus, in the presence of a genetic defect in the fibrinolysis system (the presence of PAl-1 polymorphism) with 
an initial increase in the level of PAI-1 in the blood to $25 \%$, taking $\mathrm{COC}$ significantly increases the risk of thrombotic complications.

When taking COCs, an increase in the activity of most blood procoagulants is observed, including fibrinogen, vitamin $\mathrm{K}$ dependent coagulation factors: prothrombin, VII, IX, X, XII factors. The degree of increase in the content of these factors directly depends on the dose of estrogen in the drug, and their isolated increase indicates the presence of hypercoagulation in the absence of signs of activation of intravascular coagulation and thrombosis [18].

With prolonged use of COCs, a decrease in the concentration of natural blood anticoagulants is observed: protein $C$, protein $S$, AT III. It was found that by the 3rd month of taking COCs their level does not change significantly, but by the 9th month there is a significant decrease in the anticoagulant components of the hemostasis system [19]. In addition, women taking COCs show a decrease in sensitivity to activated protein $C$. The degree of acquired resistance to activated protein C (APC-R) is similar to that in the presence of a heterozygous form of Leiden factor $V$ mutation. But unlike genetically determined APC-R, acquired APC$\mathrm{R}$ is not detected after discontinuation of this category of drugs. It is believed that the degree of APC-R in women with a heterozygous form of Leiden factor $V$ mutation taking COCs is similar to that for homozygous factor V Leiden mutation [20].

Thus, in the case of taking COCs and the presence of congenital defects of the hemostatic system, and especially combined forms of thrombophilia, there is a unidirectional effect on the hemostatic system, therefore COCs in many cases are a trigger factor for the development of thrombotic complications in case of existing imbalances in the pro- and anticoagulant mechanisms.

Nevertheless, the severity of changes in the hemostatic system caused by COCs directly depends on the concentration of estrogen and progestogen components in the drug. A correlation of the risk of thrombotic complications with the concentration of $E E$ in the drug was detected immediately after the first high-dose COCs entered the market. Subsequently, as a result of reducing the dose of estrogen by 5 times, the risk of thrombosis decreased by 4 times. Further, for many years scientists studied the effects of different gestagen components in the composition of COCs on the hemostatic system [21].

In women taking COCs before pregnancy, the majority of complications occur in the I trimester: non-developing pregnancy for a period of 5 to 7 weeks in $75 \%$, of which $40 \%$ are anembryonia, an undeveloped pregnancy from 8 to 12 weeks - in $10 \%$, spontaneous miscarriage from 5 up to 12 weeks - in $15 \%$. In the II trimester, spontaneous miscarriage after 12 weeks is $5 \%$, non-developing pregnancy after 12 weeks - 10\%. In the III trimester, late complications develop: antenatal fetal death (15\%), preeclampsia (5\%), placental insufficiency $(5 \%)$, intrauterine growth retardation (2.5\%). The majority of women with such pregnancy complications after taking COCs during the examination show congenital and / or acquired thrombophilia with the same frequency as in the population [22].

Thus, before the appointment of COCs, it is necessary to diagnose genetic and acquired forms of thrombophilia, as well as a detailed clarification of the family and personal thrombotic and obstetric history in order to identify women at risk of thrombotic complications. Considering the effect of $\mathrm{COC}$ administration on individual parts of the hemostasis system and coagulation background in general in healthy women, constant monitoring of the parameters of the hemostasis system is necessary both before the appointment of COCs and in the process of taking them. When planning pregnancy, you should stop taking COCs for 2-3 months.

Considering the effect of $\mathrm{COC}$ administration on individual parts of the hemostasis system and coagulation background in general in healthy women, constant monitoring of the parameters of the hemostasis system is necessary both before the appointment of COCs and in the process of taking them.

When prescribing hormonal contraceptive drugs, especially for women at risk of thrombotic complications, it is necessary to choose drugs that have the least effect on the hemostasis system. Constant dynamic monitoring of the indicators of the main links of the hemostasis system is recommended, depending on the identified mutations in the presence of thrombophilia in patients.

Women with family history and personal thrombotic history, as well as obstetric history, are not recommended to take COCs. If there are indications for taking COCs, dynamic monitoring of the parameters of the hemostatic system should be carried out and, if a prothrombotic condition is detected, anticoagulant therapy should be recommended. When planning pregnancy, you should stop taking COCs for 2-3 months.

Pregravid preparation should be carried out within 2-3 months before pregnancy. Activation of the hemostasis system during the $\mathrm{COC}$ flow test is an indication for prophylactic complex antithrombotic therapy with low molecular weight heparins, antiplatelet agents (if indicated), omega-3 polyunsaturated fatty acids, folic acid, magnesium and B vitamins [23].

\section{Features of the hemostatic system during physiological pregnancy and with hormonal support}

During pregnancy, the rheology of blood and its coagulation properties change significantly, which is due to hormonal changes in the body of a woman. Pregnancy affects the regulation of blood coagulation mainly due to an increase in estrogen levels, which leads to an increase in thrombin production and an increase in blood coagulation. The risk of deep vein thrombosis during pregnancy is 5 times higher than in non-pregnant women; this is due to an increase in venous stasis in the lower limbs and pelvic organs, as well as a prothrombotic change in the balance between the coagulation and fibrinolytic systems. It is believed that a change in the coagulation properties of blood plays a major role in maintaining adequate blood flow in the mother-placenta-fetus system and helps to reduce blood loss during childbirth.

Laboratory investigations of primary hemostasis include evaluating the level of von Willebrand factor (WF), platelet count, bleeding time, and platelet aggregation in vitro. Standard screening for secondary hemostasis includes the determination of prothrombin time (PT), activated partial thromboplastin time (APTT), partial thromboplastin time (PTT), and fibrinogen level. Fibrinolytic activity is judged by the level of fibrin / fibrinogen degradation products, primarily D-dimer [24].

In healthy pregnant women, there is a tendency to hypercoagulation due to an increase in the synthesis of WF, V, VII, VIII, IX, X factors, an increase in the level of fibrinogen and activation of thrombin, and a decrease in protein $S$. There is evidence of an increase in the level of coagulation factor activation markers during pregnancy, however there is no evidence that an increase in the concentration of coagulation activation markers 
precedes thrombotic complications in healthy pregnant women [25].

It has been shown that changes in the concentration of coagulation factors during pregnancy are similar to those that occur in women using COCs. This confirms the relationship between sex hormones and the hemostatic system [26].

The onset of pregnancy resulting from the implementation of the in vitro fertilization (IVF) and embryo transfer (ET) program is characterized by hormonal imbalance, which is caused by exogenous drug loading used in the ovulation stimulation protocol and support in early pregnancy. As a result, all the initial processes of adaptation of the mother's body and the formation of the fetoplacental system occur against the background of nonphysiological concentrations of estrogen and progesterone.

The frequency of venous thrombosis during the implementation of the IVF-ET program, according to various estimates, is $0.08-0.11 \%$ of all treatment cycles. The magnitude of this risk is similar to the magnitude of the risk of venous thrombosis during pregnancy and represents at least a 10 -fold increase in the underlying risk of venous thromboembolic events in women of reproductive age [27].

Investigations of the hemostatic system performed in groups of patients with controlled stimulation of ovulation in IVF-ET programs showed that with an increase in the concentration of estradiol in the blood, levels of procoagulant factors (fibrinogen, WF, factors VIII, V) increase and platelet count decreases, antithrombin, proteins $\mathrm{C}$ and $\mathrm{S}$ activity decrease, APC-R increases and the level of fibrinolysis factors tPA, PAl-1 decreases [28].

$\mathrm{PT}, \mathrm{PTT}$, and APTT tend to decrease with increasing gestational age. PT and APTT reach their minimum values at 21-27 weeks of gestation, while PTT reach their minimum values at 28-34 weeks. Evaluation of PT, APTT and PTT showed that changes in pregnancy dynamics as a result of using IVF-ET programs are identical to changes in spontaneous pregnancy [28].

Normal pregnancy as a hypercoagulable state is associated with an increase in the level of various coagulation factors, including WF and fibrinogen, as well as with an increase in the synthesis and activity of thrombin. The level of fibrinogen increases by the 20th week of spontaneous pregnancy, exceeding the initial level by 1.6 times by the end of pregnancy. The increase in WF level by the end of the I trimester reaches $46 \%$, and by childbirth $-150 \%$. As with spontaneous pregnancy, in patients with pregnancy after IVF-ET, there is an increase in the level of WF and fibrinogen in blood plasma in the dynamics of the gestational process. However, the severity of these changes varies. In the I trimester of IVF pregnancy, the level of both factors is significantly higher than with spontaneous pregnancy at the same time: the level of fibrinogen is 1.2-1.3 times higher, WF -1.4 times. This is probably a consequence of the increased level of sex hormones in the I trimester in patients with IVF pregnancy. Despite the fact that the absolute average values of fibrinogen during IVF pregnancy at 21-34 weeks of gestation remain at a higher level than during spontaneous pregnancy, they do not go beyond the reference intervals [29].

The concentration of D-dimer continuously increases throughout pregnancy and reaches maximum values (up to 2.1-2.3 $\mu \mathrm{g} / \mathrm{ml}$ ) in the III trimester. In pregnancy after IVF-ET, the maximum values exceeding the initial level by 6 times are observed already in the first weeks of pregnancy, but after 20 weeks and until birth, the level of D-dimer is comparable to that in spontaneous pregnancy [30].

Values of AT III and protein C are lower than in non-pregnant women and remain stable throughout pregnancy. The dynamics of changes in the level of AT III in IVF patients coincides with that in spontaneous pregnancy [30].

\section{Conclusion}

The scientific concept of this work is different in that we have summarized and analyzed information on the effect of sex hormones entering the woman's body from the outside on individual links and the hemostatic system as a whole, determined the relationship between drug administration and thrombotic complications, optimized the sequence of diagnosis of conditions predisposing to thrombosis.

The scientific novelty of this work is that we have compared the effects of COCs and hormonal changes during pregnancy on the hemostatic system and found that changes in the hemostatic system in women taking COCs are similar to those that occur during pregnancy. So according to the literature, the features of the hemostatic system in patients taking COCs correspond to those in the physiological course of pregnancy. Comparison of the dynamics of the main laboratory indices of spontaneous pregnancy and pregnancy resulting from the implementation of the IVF-PE program shows the absence of significant differences that would require the development of a specific algorithm for monitoring uncomplicated induced pregnancy.

\section{Conflict of interest}

The authors declare that they have no conflict of interest

\section{References}

1. James $\mathrm{AH}$, Konkle BA, Bauer KA. Prevention and treatment of venous thromboembolism in pregnancy in patients with hereditary antithrombin deficiency. Int J Womens Health 2013; 5: 233-241. https://doi.org/10.2147/ijwh.s43190.

2. Tepper NK, Boulet SL, Whiteman MK, Monsour M, Marchbanks PA Hooper WC, et al. Postpartum venous thromboembolism: incidence and risk factors. Obstet Gynecol 2014; 123(5): 987-996. https://doi.org/10.1097/aog.0000000000000230.

3. van Hylckama Vlieg A, Helmerhorst FM, Vandenbroucke JP, Doggen CJ, Rosendaal FR. The venous thrombotic risk of oral contraceptives, effects of oestrogen dose and progestogen type: results of the MEGA case-control study. BMJ 2009; 339: b2921. https://doi.org/10.1136/bmj.b2921.

4. van Vliet HA, Frolich $M$, Christella $M$, Thomassen LG, Doggen CJ, Rosendaal FR, et al. Association between sex hormone-binding globulin levels and activated protein $C$ resistance in explaining the risk of thrombosis in users of oral contraceptives containing different progestogens. Hum Reprod 2005; 20(2): 563-568. https://doi.org/10.1093/humrep/deh612.

5. Lidegaard $\varnothing$, Edström B, Kreiner S. Oral contraceptives and venous thromboembolism: a five-year national case-control study. Contraception 2002; 65(3): 187-196. https://doi.org/10.1016/s00107824(01)00307-9.

6. Lidegaard $\varnothing$, Nielsen LH, Skovlund CW, Skjeldestad FE, Løkkegaard E. Risk of venous thromboembolism from use of oral contraceptives containing different progestogens and oestrogen doses: Danish cohort study, 2001-9. BMJ 2011; 343: d6423. https://doi.org/10.1136/bmj.d6423. 
7. Dinger J, Assmann A, Möhner S, Minh TD. Risk of venous thromboembolism and the use of dienogest- and drospirenonecontaining oral contraceptives: results from a German case-control study. J Fam Plann Reprod Health Care 2010; 36(3): 123-129. https://doi.org/10.1783/147118910791749416

8. Dinger JC, Heinemann LA, Kühl-Habich D. The safety of a drospirenonecontaining oral contraceptive: final results from the European Active Surveillance Study on oral contraceptives based on 142,475 womenyears of observation. Contraception 2007; 75(5): 344-354. https://doi.org/10.1016/j.contraception.2006.12.019.

9. Keren-Politansky A, Breizman T, Brenner B, Sarig G, Drugan A. The coagulation profile of preterm delivery. Thromb Res 2014; 133(4): 585589. https://doi.org/10.1016/j.thromres.2014.01.018.

10. Adler G, Mahmutbegovic E, Valjevac A, Adler MA, Mahmutbegovic N, Safranow K, et al. Association Between - 675 ID, 4G/5G PAl-1 Gene Polymorphism and Pregnancy Loss: A Systematic Review. Acta Inform Med 2018; 26(3): 156-159. https://doi.org/10.5455/aim.2018.26.156159.

11. Dorgalaleh A, Rashidpanah J. Blood coagulation factor XIII and factor XIII deficiency. Blood Rev 2016; 30(6): 461-475. https://doi.org/10.1016/j.blre.2016.06.002.

12. Hiraoka M, Kagawa Y. Genetic polymorphisms and folate status. Congenit Anom (Kyoto) 2017; 57(5): 142-149. https://doi.org/10.1111/cga.12232.

13. Szelenberger R, Kacprzak M, Bijak M, Saluk-Bijak J, Zielinska M. Blood platelet surface receptor genetic variation and risk of thrombotic episodes. Clinica Chimica Acta 2019; 496: 84-92. https://doi.org/10.1016/j.cca.2019.06.020.

14. Mina A, Favaloro EJ, Mohammed S, Koutts J. A laboratory evaluation into the short activated partial thromboplastin time. Blood Coagul Fibrinolysis 2010; 21(2): 152-157. https://doi.org/10.1097/mbc.0b013e3283365770.

15. Milovanov AP, Kuznetsova NB, Fokina TV. Role of Immune Distribution of Tissue Factor in the Development of Hemostasis during the First Trimester of Normal Pregnancy. Bull Exp Biol Med 2019; 166(4): 503506. https://doi.org/10.1007/s10517-019-04382-7.

16. Huynh A, Arnold DM, Moore JC, Smith JW, Kelton JG, Nazy I. Development of a high-yield expression and purification system for platelet factor 4. Platelets 2018; 29(3): 249-256. https://doi.org/10.1080/09537104.2017.1378808.

17. Küçük $M$, Sezer SD, Odabaşi $A R$, Güner Z, Yuksel H, Serter $M$. The effect of low-dose combined oral contraceptive containing 100 ug levonorgestrel on plasma plasminogen activator inhibitor-1 concentrations. Clin Exp Obstet Gynecol 2011; 38(1): 54-56. https://pubmed.ncbi.nlm.nih.gov/21485727.

18. Trenor CC 3rd, Chung RJ, Michelson AD, Neufeld EJ, Gordon CM, Laufer MR, et al. Hormonal Contraception and Thrombotic Risk: A Multidisciplinary Approach. Pediatrics 2011; 127(2): 347-357. https://doi.org/10.1542/peds.2010-2221.

19. Kovac MK, Lalic-Cosic SZ, Dmitrovic JM, Djordjevic VJ, Radojkovic DP. Thrombin generation, D-dimer and protein $\mathrm{S}$ in uncomplicated pregnancy. Clin Chem Lab Med 2015; 53(12): 1975-1979. https://doi.org/10.1515/cclm-2014-1030.

20. Hale SA, Sobel B, Benvenuto A, Schonberg A, Badger GJ, Bernstein IM. Coagulation and Fibrinolytic System Protein Profiles in Women with Normal Pregnancies and Pregnancies Complicated by Hypertension.

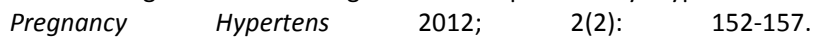
https://doi.org/10.1016/j.preghy.2012.01.004.

21. Alijotas-Reig J, Palacio-Garcia C, Llurba E, Vilardell-Tarres M. Cellderived microparticles and vascular pregnancy complications: a systematic and comprehensive review. Fertil Steril 2013; 99(2): 441449. https://doi.org/10.1016/j.fertnstert.2012.10.009.

22. Ataullakhanov FI, Koltsova EM, Balandina AN, Serebriyskiy II, Vuimo TA, Panteleev MA. Classic and Global Hemostasis Testing in Pregnancy and during Pregnancy Complications. Semin Thromb Hemost 2016; 42(7): 696-716. https://doi.org/10.1055/s-0036-1592303.

23. Ozay AC, Emekci Ozay O, Okyay RE, Cagliyan E, Kume T, Gulekli B. Different Effects of Myoinositol plus Folic Acid versus Combined Oral Treatment on Androgen Levels in PCOS Women. Int J Endocrinol 2016; 2016: 3206872. https://doi.org/10.1155/2016/3206872.

24. Palta S, Saroa R, Palta A. Overview of the coagulation system. Indian J Anaesth 2014; 58(5): 515-523. https://doi.org/10.4103/0019$\underline{5049.144643}$.

25. Rühl H, Berens C, Winterhagen FI, Reda S, Müller J, Oldenburg J, et al. Increased Activated Protein C Response Rates Reduce the Thrombotic Risk of Factor V Leiden Carriers But Not of Prothrombin 20210G >A Carriers. Circ Res 2019; 125(5): 523-534. https://doi.org/10.1161/circresaha.119.315037.

26. Hedengran KK, Andersen MR, Stender S, Szecsi PB. Large D-Dimer Fluctuation in Normal Pregnancy: A Longitudinal Cohort Study of 4,117 Samples from 714 Healthy Danish Women. Obstet Gynecol Int 2016; 2016: 3561675. https://doi.org/10.1155/2016/3561675.

27. Gurunath S, Vinekar S, Biliangady R. Assisted Reproductive Techniques in a Patient with History of Venous Thromboembolism: A Case Report and Review of Literature. J Hum Reprod Sci 2018; 11(2): 193-197. https://doi.org/10.4103/jhrs.jhrs $58 \quad 17$.

28. Bagot CN, Leishman E, Onyiaodike CC, Jordan F, Freeman DJ. Normal pregnancy is associated with an increase in thrombin generation from the very early stages of the first trimester. Thromb Res 2017; 157: 4954. https://doi.org/10.1016/i.thromres.2017.06.027.

29. Singh S, Rishi B, Sharma S. Shortened coagulation profile in pregnancy - comparative analysis in different trimesters - retrospective study from a tertiary care centre. EJPMR 2016; 3(11): 548-551. https://www.ejpmr.com/home/abstract_id/1723.

30. Qin J, Liu X, Sheng X, Wang H, Gao S. Assisted reproductive technology and the risk of pregnancy-related complications and adverse pregnancy outcomes in singleton pregnancies: a meta-analysis of cohort studies. Fertil Steril 2016; 105(1): 73-85. https://doi.org/10.1016/j.fertnstert.2015.09.007.

\section{Authors:}

Evgeniya V. Kirakosyan - PhD student, Department of Obstetrics, Gynecology, Perinatology and Reproductology, I.M. Sechenov First Moscow State Medical University (Sechenov University), Moscow, Russia. https://orcid.org/0000-0002-6021-2449.

Elena A. Sosnova - MD, PhD, professor, Department of Obstetrics and Gynecology №1, I.M. Sechenov First Moscow State Medical University (Sechenov University), Moscow, Russia. https://orcid.org/0000-0002-1732$\underline{6870}$. 